\title{
Initial linking methods and their classification
}

\author{
Leif Kahl Kristensen ${ }^{1}$ \\ ${ }^{1}$ Department of Physics and Astronomy, University of Aarhus, DK-8000 Aarhus C, Denmark \\ email: LKK@PHYS.AU.DK
}

\begin{abstract}
The problem of initial linking of asteroids is of increasing interest for the next generation surveys. During the first week after discovery elliptical elements are very uncertain and other methods are used. A summary is given of 7 initial linking methods. There are two different types: In one, a search area is computed on a second night from the known and undoubtedly linked positions, typically on the first night. The other type assumes candidates which are then checked by the computation of $\mathrm{O}-\mathrm{C}$ residuals of an orbit. Computations may be classified as belonging to the 3 -dimensional space or the 2-dimensional sky-plane. A new basis, with a simpler computational algorithm, is given for the widely used Väisälä method. For a new N-Observation Orbit method a simple, efficient PC-programme is given.
\end{abstract}

Keywords. Orbit determination; identification; ephemerides

\section{Introduction}

Different methods can be used for linking a great number of asteroids during their first critical lunation. When 3-4 nights of observations are secured during 2-3 weeks it makes sense to compute elliptical elements but different methods are needed at start. Here we try to list the 7 known methods but will not give exhaustive references. For familiar methods we only give remarks not available elsewhere.

Focus is on computational efficiency, so statistical (Monte Carlo) methods based on many orbits are excluded in advance.

There are essentially two problems:

1) Some observations are given for a definite object but not enough for the determination of an orbit. A typical example is $1-5$ observations on a single night. The problem is to predict a search area another night in which the candidates may be found.

2) Two groups of observations are given which may possibly belong to the same object. Typically two nights with daily motions. The problem is to check if an orbit exists which gives $\mathrm{O}$ - $\mathrm{C}$ residuals consistent with observational errors.

Computing methods are of two types:

A) Computing takes place in 3-dimensional space. Topocentric coordinates (X,Y,Z) of the observer is for instance used.

B) All computations occur in the 2-dimensional sky-plane. These methods are based on the smoothness of the apparent motion and use interpolation formulae.

In this scheme the widely used (generalized) Väisälä method is classified 1A and some aspects are discussed in Sec. 7.

Type 1B methods are: extrapolation, (T,I)-coordinates and distant objects, to be discussed in Sec. 2, 3 and 4.

Type $2 \mathrm{~B}$ is $\mathrm{L}-\mathrm{R}$ method and daily motion method in Sec. 5 and 6 .

Type $2 \mathrm{~A}$ is the N-Observation method discussed in Sec. 8. Its use for impact monitoring is given in Sec. 9. A (Pascal) PC programme is given in the Appendix. 


\section{Extrapolation (Type 1B)}

To link the positions each individual night we "predict" second positions at $t_{2}=t_{1}+h$ from the first at $t_{1}$ by the local mean daily motion and its scatter. For main belt objects near opposition we have the daily motions in ecliptical longitude and latitude:

$$
\lambda^{\prime}=0.225 \pm 0.0315^{\circ} / d, \quad \beta^{\prime}=0 \pm 0.045^{\circ} / d
$$

With $h=1 / 24 d$ the area to be searched is $1.1 \times 10^{-5}$ sq. deg. Assume a density of objects $\mu=900$ per square degree which corresponds to a mean distance $1^{\prime}\left(=\frac{1}{2} / \sqrt{\mu}\right)$ to the nearest neighbour. The probability to find two objects, the correct one plus a random straggler, is only about $1 \%$. The moving objects are assumed distinguished from the much larger density of stars by trailed images. There seems to be little difficulty at this first step.

The following nights objects are obtained by linear extrapolation based on Lagrange formula with remainder term (Abramowitz \& Stegun 1970, 25.2.1):

$$
f(t)=f\left(t_{1}\right) \frac{t_{2}-t}{t_{2}-t_{1}}+f\left(t_{2}\right) \frac{t-t_{1}}{t_{2}-t_{1}}+\left(t-t_{1}\right)\left(t-t_{2}\right) \frac{f^{\prime \prime}(\xi)}{2}
$$

Here $t_{1} \leqslant \xi \leqslant t$ if $t_{1} \leqslant t_{2} \leqslant t$. The remainder term can be estimated from the second differences $\Delta^{2}$ in the 10-days ephemerides, conveniently in EMP1988 (the last volume stating differences). A sample gave typical values of $\Delta^{2} \alpha$ to be of order $-2^{m}$ before and $+2^{m}$ after opposition. Similarly $\Delta^{2} \delta \approx \pm 15^{\prime}$. After 5 days the area to be seached is thus $1 / 8^{\circ}$ by $1 / 16^{\circ}$. The observational error $\pm \sigma$ (here assumed $0.15^{\prime \prime}$ ) gives

$$
\pm \sigma \frac{\sqrt{\left(t-t_{1}\right)^{2}+\left(t-t_{2}\right)^{2}}}{t_{2}-t_{1}}
$$

With $\frac{1}{2}$ hour between exposures this gives after 5 days $\pm 0.8^{\prime}$.

All interpolation methods are affected by the short-period parallax which gives an error in the daily motion of order

$$
\cos \delta \alpha^{\prime}=-2 \pi \frac{8^{\prime \prime} 80}{\Delta}
$$

where $\Delta$ is the geocentric distance. For main belt objects $1 / \Delta=0.75 \pm 0.36$. After 5 days there is a systematic error $-3.5^{\prime} \pm 1.7^{\prime}$ in $\alpha$. The corresponding effect in $\delta$ is negligible. Adding the above errors $\left( \pm 3.8^{\prime}\right.$ from $(2), \pm 0.8^{\prime}$ from (3) and $\pm 1.7^{\prime}$ from (4)) gives the uncertainty in $\alpha$ : $\pm 4.2^{\prime}$. Similarly in $\delta$ adding $\pm 1.9^{\prime}$ from (2) and $\pm 0.8^{\prime}$ from (3) gives $\pm 2.1^{\prime}$. The $1 \sigma$ limit area is thus 0.0098 square degree, corresponding to an average of 8.8 erroneous objects. Including the object in question there are around 10 candidates for which trial orbits must be found. The example illustrates the relative importance of the approximations which scales like $T^{4}, T^{2}$ and $\mathrm{T}$ respectively.

\section{3. $(\mathrm{T}, \mathrm{I})$ coordinates (Type:1B)}

If we assume circular orbits with $a=2.64 \mathrm{AU}$ and longitude in orbit $90^{\circ}$ we can compute the inclination (I) and the opposition date (T) for any single position. This corresponds to a coordinate transformation from $(\alpha, \delta)$ to $(\mathrm{T}, \mathrm{I})$ (Kristensen 1990). For the "mean" main belt asteroid $\mathrm{T}$ and I are constant and for the neighboring real asteroids $\mathrm{T}$ and I are slowly varying and the mean of their daily motion relative to the center of the belt is zero. There is thus no mean motion depending on the position in the sky as the constants in the ordinary coordinates in (1). The main irregularities in the apparent 
motion is caused by the reflex motion of the Earth which is much reduced and the retrograde loop entirely removed. The parallax is partly corrected for to the mean distance to the main belt. The method has the same accuracy as Väisälä's perihelion orbits with $q=a$ and gives an alternative justification of this method. Väisälä assumes essentially, as did Thiele (1883), a smooth motion on a sphere with radius q. In (T,I) the computations are easier and based on individual rather than pairs of observations. Each observation $(\alpha, \delta)$ is transformed to the slowly varying $(\mathrm{T}, \mathrm{I})$. This makes interpolation methods more efficient. A trial with 4 synthetic plates with a density $\mu=900$ objects/deg.sqr. (Kristensen 2005, unpublished) has shown that single main belt positions each night can be effectively linked. Only algorithms well developed in computer science are needed, such as finding all objects in given search areas, combining positions and checking differences.

\section{Distant objects (Type:1B)}

In classical stellar astrometry the elements are position at the epoch, proper motion, radial velocity and the (yearly) parallax. Compared with orbit determination for main belt asteroids this is much simplified! Surprisingly, the transition region between these two methods is rather near, say at 20 AU. For a few years the simple method ignoring acceleration and using only plate coordinates may be used for TNO's (Kristensen 2004).

\section{L-R methods (2B)}

Assume 4 positions at times: $-\frac{1}{2} h,+\frac{1}{2} h, T-\frac{1}{2} h$ and $T+\frac{1}{2} h$. Use the formulae 25.1.5 and 25.1.10 in Abramowitz \& Stegun (1970) and re-arrange, this gives:

$$
\frac{f\left(t_{2}\right)(T+h)-f\left(t_{1}\right)(T-h)}{2 h}+\frac{f\left(t_{4}\right)(T-h)-f\left(t_{3}\right)(T+h)}{2 h}=\frac{T\left(T^{2}-h^{2}\right)}{12} f^{(3)}(\xi)
$$

The first term is linear extrapolation from the first night to $T / 2$ and the second term extrapolates the second night to the same instant. From the first and second nights we construct a fictitious plate at $T / 2$ on which adjoining objects can be identified.

This method is a special case of the L-R method (Kristensen 1992). It is denoted the "midpoint" method by Kubica (2005) who tested it. The remainder term can be estimated by the third differences of the 10-days ephemerides. For $T=7$ the error is of order $0.5^{\prime}$. In $\alpha$ there is still the systematic parallax displacement. The search area scales as $T^{6}$.

\section{Daily motion integration (Type 2B)}

To check if observations a second night (at $t=T$ ) belong to those the first night (at $t=0)$ the daily motions must agree. The numerical integration of, say $d \delta(t) / d t$, gives (Abramowitz \& Stegun 1970, 25.4.1)

$$
\int_{0}^{T} \delta^{\prime}(t) d t=\delta(T)-\delta(0)=\frac{T}{2}\left(\delta^{\prime}(T)+\delta^{\prime}(0)\right)-\frac{T^{3}}{12} \delta^{(3)}(\xi)
$$

from which

$$
\delta^{\prime}(T)=\frac{2(\delta(T)-\delta(0))}{T}-\delta^{\prime}(0)+\frac{T^{2}}{6} \delta^{(3)}(\xi)
$$

As an example (the main belt object (2156) Kate in 2002, Appendix B, Table 1). 
Table 1. Daily motion for object (2156) Kate

\begin{tabular}{cc}
\hline \multicolumn{1}{c}{$t$} & $\delta$ \\
\hline-0.05 & $11.040279^{\circ}$ \\
0.05 & $11.044407^{\circ}$ \\
6.95 & $11.276349^{\circ}$ \\
7.05 & $11.278964^{\circ}$ \\
\hline
\end{tabular}

This gives the daily motion $\delta^{\prime}(0)=+0.041280^{\circ} / d$ and from the 7 -days interval +0.033616 . By $(7)$ is obtained $\delta^{\prime}(7.0)=+0.025952^{\circ} / d$ to be compared with the true value +0.026150 , the error is $O-C=+0.000198$. The difference between the daily motions of two objects taken at random $\pm 0.070^{\circ} / d$ due to the scatter (1) from the mean motion. It is thus unlikely that the two nights refer to different objects. Formula (7) is equivalent to (5) in the limit $h \rightarrow 0$.

\section{The generalized Väisälä method (Type 1A)}

This well known method gives search areas from two observations under different assumptions, partial knowledge or a priori information. Marsden (ACM 1991, 395) assumes limits on eccentricity e, semimajor axis a and peri- and aphelion distances ( $\mathrm{q}$ and Q). Kristensen (2002) assumes intervals for the geocentric distance $(\Delta)$ and $r^{\prime}=d r / d t$. Milani et al. (2005) assume $\mathrm{r}$ and $r^{\prime}$ limited by energy. We shall not comment on these methods but only show, that the demarcation line of the search area can be delineated very simply for main belt objects at opposition by only one single Väisälä perihelion orbit.

Formula (5) in Kristensen (2002) shows that there are two non-linear terms in the apparent motion. One is due to the attraction of the Sun and, because $r^{3}>R^{3}$, is proportional to $1 / \Delta$. The other is a perspectivistic effect along the daily motion. In Fig. 1 we denote by $\mathrm{V}$ the position by assuming $\Delta=1.0$ and $r^{\prime}=0$. The position $\mathrm{L}$ is obtained by linear extrapolation along the great circle between position 1 and 2 and corresponds to $\Delta \rightarrow \infty$. The perihelion orbits are approximately on the line connecting $\mathrm{L}$ and $\mathrm{V}$ and subdivided as $1 / \Delta$. For constant values of $\Delta$ the curves of varying $r^{\prime}$ are situated on great circles through position 2 and along the direction of motion. For $\Delta=1$ and $r^{\prime}=k e / r \approx 0.0024$ (assuming $e=0.20$ ) formula (5) in Kristensen (2002), the "foreshortening" term, is a product of the motion from the Nov. 30.94 position (2) to position 3 at Nov. 57.76 and the factor $-\Delta^{\prime} t / \Delta \approx-t r^{\prime} / \sqrt{1+\Delta}$ :

$$
\left(-5.4^{\circ},+1.3^{\circ}\right) \times(-16.82) \times 0.0024=\left(+0.22^{\circ},-0.05^{\circ}\right)
$$

The three lines of constant $r^{\prime}$ in the figure are great circles through position 2 .

A sphere with radius $q$ osculates perihelion orbits and to a good approximation the motion is uniform. Thiele (1883) assumed uniform great circle motion on this sphere. There is thus little difference between these methods for small arcs.

\section{N-Observation Orbits (Type 2A)}

The importance of using all available observations for the initial orbit is stressed by Branham (2005). The first and last positions are urgent to increase the arc but any number in between improves the critical geocentric distance $\Delta$ - especially by parallax 


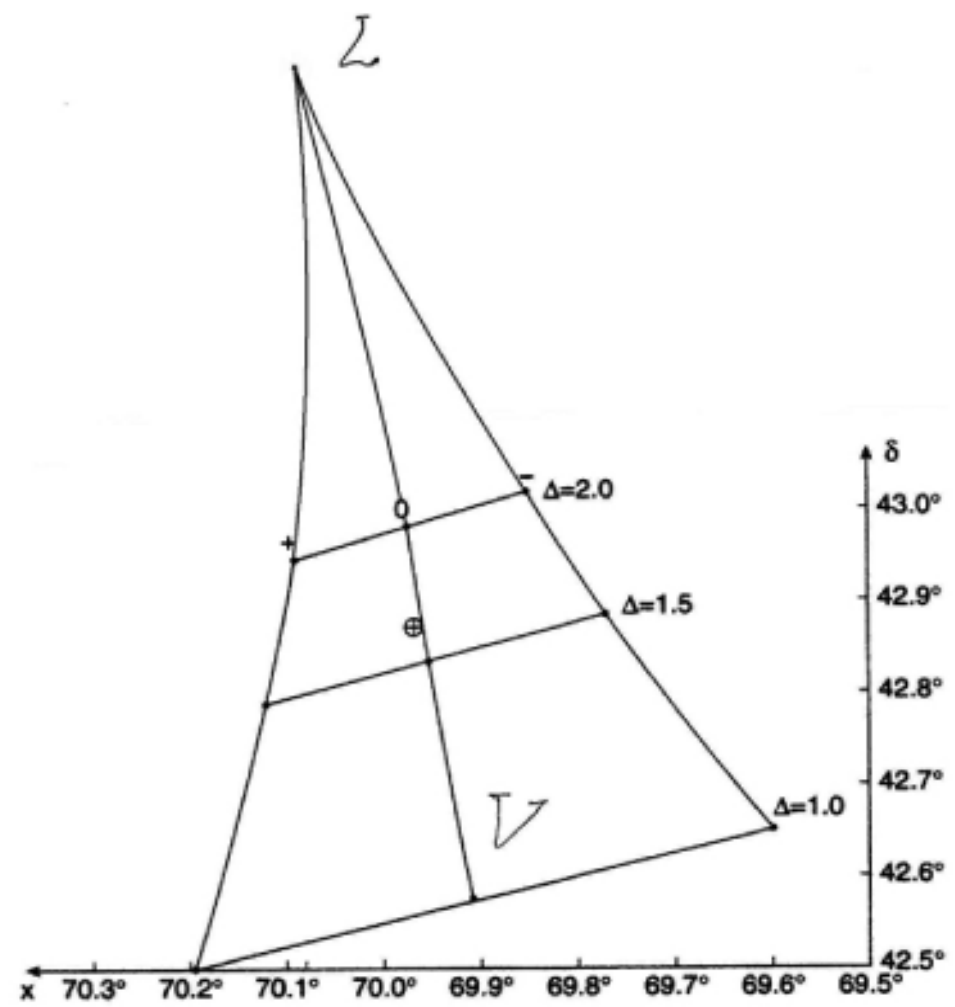

From the two discovery positions of 1984 WH Nov. 25.95 and 30.94 (MPC 9263, 9326) the recovery position on Dec. 17.76 is predicted by assuming $\Delta=1.0,1.5$, and 2.0. Trial values for $r^{\prime}$ were 0 and $\pm 0.20 / \sqrt{1+\Delta}$. The $r^{\prime}$ variation lines are segments of great circles all passing through the first position as a "radiant." This is a perspectivistic effect of the velocity being parallel to $n_{1}$ by Eq. (38). The search area is limited by curves corresponding to receeding $\left(r^{\prime}>0\right)$ and approaching $\left(r^{\prime}<0\right)$ heliocentric motion. Midpoints give the Varisalla (1939) variation curve of perihelion orbits $\left(r^{\prime}=0\right)$. The area is only 0.13 square degrees. It is indicated by the six points which can easily be computed by a programmable pocket calculator. The object happened to be near perihelion and was actually found at the indicated point near the Vatisala line of variation.

Figure 1. Search area from two positions. The vertex $\mathrm{L}$ is obtained by linear extrapolation and corresponds to $\Delta=\infty$ and $\mathrm{V}$ assumes $\Delta=1.0$ and $r^{\prime}=0$. The three lines of variation of $\mathrm{r}^{\prime}$ are great circles through the first position (see text). The Väisälä locus connects L and V and is subdivided as $1 / \Delta$. The search area is essentially determined by one single perihelion orbit

from different sites. The present method (Kristensen 2005) gives positions as a strictly linear function of the coordinates $\mathrm{x}$ and velocities $x^{\prime}$ at the epoch To in terms of the $\mathrm{f}$ and $\mathrm{g}$ coefficients. Good approximations for $\mathrm{f}$ and $\mathrm{g}$ are obtained by a trial value for the heliocentric distance $\mathrm{r}$. Adopting $r=2.30$ gives 3-4 correct figures for main belt objects during the first month, - the shorter the arc the better the approximation! Given $\mathrm{f}$ and $\mathrm{g}$ each observation gives stricly linear equations of condition which are adjusted rigorously by a least squares solution for the elements $\mathrm{x}$ and $x^{\prime}$ by the classical method (Watson 1868) of Gauss elimination. We note especially that the square sum $\left(\chi^{2}\right)$ is obtained before the solution which may be skipped if a statistical $\chi^{2}$-test indicates inconsistent 
observations. Realistic observational error estimates may be obtained from Bykov et al. (2002) and Carpino et al. (2003). Regardless of any errors in $\mathrm{f}$ and $\mathrm{g}$ the O-C residuals by 3 observations are exactly zero. For observations on 3 nights the daily motion has the 3-4 figure accuracy. This makes the method very suitable for the initial linking problem. Due to the linear equations there is always a single solution and no need for differential corrections. It may, however, be necessary to iterate $r=|\mathbf{x}|$, especially if four nights are observed.

My first step towards this method was the projection matrix (18) in Kristensen (2002) but I could not reduce the right hand sides of the normal equation before I noted the ingenious trick of T.N. Thiele's "fabricated observations" (Thiele 1889). After submission of my paper (Kristensen 2005) I found a similar method by Neutsch (1981). Some advantages of these methods are: all available observations are used from the start, two simultaneous positions from different sites gives $\Delta$ by parallax, Radar surveillance observations can be included, the intermediate step of differential corrections of elliptical elements is not used so the possible lack of convergence in this step is avoided, computations are strictly linear in degenerate cases and convergence problems are avoided, within 2-3 weeks for main belt asteroids iterations are not necessary, which is especially useful for initial linking and, finally, impact monitoring is facilitated.

Rather than explaining how the coefficients of the normal equations are derived is here given a simplified PC-programme in Pascal in the Appendix.

1) The main belt object (2156) Kate observed 2 nights at times $J D=2452200.5+$ $t$. Maximum $\mathrm{O}-\mathrm{C}$ is -0.005 " so the two short arcs are undoubtedly linked. The advantage of an initial orbit based on 4 - rather than 3 - observations is obvious.

2) The Centaur (60558) $2000 \mathrm{EC}_{98}$ discussed by Milani et al. (2005). There are 3 nights: Feb. 5, Mar. 3 and 4 with daily motions. The mean positions on 3 nights are always fitted exactly but it is unlikely that the motions also agree if not referring to the same object. Rounding to 4 decimals in degrees corresponds to a mean observational error \pm 0.10 ". The recovery position April 4 is predicted with errors +22.970 " and $-13.076 "$ and a motion accurate enough for identification. There is no need to repeat the computation based on $r=2.30$ with the improved value $r=13.446$, obtained from the solution: $x=(-13.07,+2.93,+1.14)$.

3) The last example is the NEO (85640) $1998 \mathrm{OX}_{4}$ at 6 ephemeris positions 2006 Aug. $24.0+t$. The first run with $r=2.30$ indicates an NEO with $r=1.20$. The orbit is well determined and the $\mathrm{f}$ and $\mathrm{g}$ coefficients should be improve by rigorous expressions, but this facility is removed from the present abbreviated programme.

\section{Impact monitoring}

If the elements are coordinates and velocities at the epoch, $\mathrm{x}$ and $x^{\prime}$, a (Radar) geocentric distance $\Delta$ or a (Doppler) radial velocity $\Delta^{\prime}$ at time $\mathrm{t}$ gives a linear equation of condition which contributes directly to the normalequations. However, these facilities have been removed from the simplified programme in the Appendix. Two positions give 4 conditions and adopting trial values for $\Delta$ and $\Delta^{\prime}$ the orbit is determined and also the coordinates $x\left(\Delta, \Delta^{\prime} ; t\right)$ et.c. Earth coordinates are $X(t)$ and a collision at time $\mathrm{T}$ gives 3 equations:

$$
x\left(\Delta, \Delta^{\prime} ; T\right)=X(T) \quad \text { etc. }
$$

for the unknowns $\Delta$ and $\Delta^{\prime}$ and $\mathrm{T}$.

Mathematical solutions of (9) are very frequent if the daily motion and $\Delta$ are small (Kristensen 2002b). Nothing about a collision risk may be said before enough observations 
have been secured to make sense for $\Delta$. The $2004 \mathrm{AS}_{1}$ false alarm was due to the indeterminateness of $\Delta$ and the adoption of a too small value.

In the case of (85640) with the 3 -days arc in Appendix B there is a close passage at $t=16$ (EMP 2006, p.680). To check if it actually is a hit we assume a Radar observation with $\Delta=0$ at $t=16$ and re-adjust. The assumption is not consistent with the optical positions. The O - C residuals are max. -0.099 " in Appendix B but is forced to 1472 ".

By series of trial times $\mathrm{T}$ it can be checked if a collision is imminent. This gives a 1-dimensional set rather than a 2-dimensional set of trial orbits.

\section{Acknowledgements}

The author is much indebted to Mikael Julian Jørgensen for the $\mathrm{LAT}_{\mathrm{E}} \mathrm{X}$ manuscript and to the referee, Giovanni F. Gronchi, for improvements of the text.

\section{References}

Abramowitz, M. \& Stegun, I. 1970, Handbook of Math. Functions, Nat. Bureau of Standards, Washington.

Branham, R. L. 2005, Celest. Mech. Dyn. Astron. 93, 53

Bykov, O. P., L'vov, V. N., Izmailov, L. S., \& Sumzina, N. K. 2002, in: Proceedings of ACM 2002, ESA-SP-500

Carpino, M., Milani, A. \& Chesley, S. 2003, Icarus 166, 248

Ephemerides of minor Planets for 1988, Institute for Theoretical Astronomy, Leningrad 1987

Kristensen, L. K. 1990, Astron. Nachr. 311, 133

Kristensen, L. K. 1992, Astron. Astrophys. 262, 606

Kristensen, L. K. 2002, Icarus 159, 339

Kristensen, L. K. 2002, in: Proceedings of ACM 2002, ESA-SP-500

Kristensen, L. K. 2004, Astron. J. 127, 2424

Kristensen, L. K. 2005, N-Observations and Radar Orbits (Submitted)

Kubica, J. 2002, Efficient discovery of spatial associations and structure, Robotic Institute, Carnegie Mellon univ., Pittsbúrgh, Pennsylvania

Marsden, B. G. 1992, in: Proceedings Asteroids, Comets and Meteors, Houston, Texas, p. 395

Milani, A. \& Knežević Z. 2005, Celest. Mech. Dyn. Astron. 92, 1

Neutsch, W. 1981, Astron. Astrophys. 102, 59

Thiele, T. N. 1889, Almindelig Iagttagelseslre. (Reitzel, Copenhagen) (In Danish, English translation in E. L. Lauritzen: Thiele-Pioneer in Statistics. Oxford Univ. Press, 2002)

Thiele, T. N. 1883, Astron. Nachr. 107, 291, 357

Thiele, T. N. 1903, Theory of Observations, Charles \& Edwin Layton, London

Watson, J. C. 1868, Theoretical Astronomy, J. B. Lippincott and Co., reprinted by Dover Publ. New York 1964

\section{Appendix A. N-Observation Orbits PC programme}

The elements are $\mathrm{x}$ and $x^{\prime}$ at epoch T0. Positions are expressed in terms of the $f \approx 1$ and $g \approx t$ coefficients. Four figure accuracy is obtained the first month by assuming $r=2.30$. Given $\mathrm{f}$ and $\mathrm{g}$ the least squares adjustment is stricly linear in the elements $\mathrm{x}$ and $x^{\prime}$. The coefficients of the normalequations are stored in a $7 \times 7$ matrix. The programme is a simplified version with Radar observations and computation of $\mathrm{f}$ and $\mathrm{g}$ from the elements by exact formulae removed. Perturbations and light-time are ignored.

The first line in input has 4 numbers: $\mathrm{N}$ observations, epoch T0, adopted $\mathrm{r}$ and observational mean error (m.e.). The following $\mathrm{N}$ lines gives times of observation, coordinates $(X, Y, Z)$ of the observatory, observed (true) RA and Decl in units of degrees, and finally a weight $\mathrm{w}$. The program stops when a first line is $-9-9-9-9$. 
The output repeats the first line in input. The next line gives the square sum of the residuals $\left(\mathrm{AO}[7,7]\right.$ or $\left.\chi^{2}\right)$ before and after a Gauss elimination and the number of degrees of freedom. The $\mathrm{N}$ lines give: $\mathrm{t}, \mathrm{w}$, observed and computed RA and Decl (in degs) and $O-C^{\prime \prime}$ (in seconds of arc).

The $1 \frac{1}{2}$ page source code in Turbo-Pascal, the executable PC programme and the sample Input/Output may be requested from the author by e-mail. 